Öfters übersetzt wurde der Hymnus auf Christus: von Fr. Münter, Über die älteste christl. Poesie (vor seiner Übersetzung der Offenb. St. Joh.) Kopenhagen 1806 S. 33 ff; von Rambach, Eylert, Schulthess in den oben S. LXXVI angeführten Werken; C. R. Hagenbach in Rheinwalds Repertorium 14 (1836) S. $114 \mathrm{f}$ und Die christl. Kirche in den ersten drei Jahrh. Leipzig 1853 S. 222 (abgedruckt bei J. Kayser, Beiträge z. Geschichte u. Erklärung der ältesten Kirchenhymnen² Paderborn 1881 I S. $28 \mathrm{f}$ und bei 0 . Bardenhewer, Geschichte der altkirchl. Literatur II Freib. i. Br. 1903 S. 32); C. Fortlage, Gesänge christl. Vorzeit Berlin 1844 S. 38-40 (abgedruckt bei A. Baumgartner, Geschichte der Weltliteratur IV Freib.i. Br. 1900 S. 18 f); J.F.H. Schlosser, Die Kirche in ihren Liedern Mainz 1852 II S. 107-109; F. Ranke in F. Pipers Evang. Kalender f. 1868 Berlin 1868 S. 29-31.

2. Französisch. Protr. wurde übersetzt von D. Cousin Paris 1684. - Nur Quis div. salv. und Paed. I-III sind enthalten in: Les Oeuvres de S. Clément d'Alexandrie traduites du Grec [par Nic. Fontaine] Paris chez Pralard 1696. - Auszüge aus allen Werken bietet D. M. N. S. Guillon, Bibliothèque choisie des pères de l'Église greeque et latine Paris 1822 I p. $375 \mathrm{ff}$. - Eine vollständige Übersetzung gab Ant. Eug. de Genoude, Les pères de l'Église IV V Paris 1839.

3. Englisch. Sämtliche Werke sind übersetzt in A. Roberts und J. Donaldson, Ante-Nicene Christian Library vol. IV. XII. XXII S. 185-217; XXIV S. 137-181 Edinburgh 1867-1872. Der Übersetzer war W. Wilson. Das Gleiche bietet der von A. Cleveland Coxe besorgte amerikanische Abdruck der englischen Sammlung: The AnteNicene Fathers II S. 171-604 Buffalo 1887. - Quis div. salv. übersetzte J. Jones (Clement, A discourse concerning the salvation of rich men London 1711) und P. Mordaunt Barnard (in der Sammlung Early Church Classics, herausgegeben von der Society for Promoting Christian Knowledge London 1901). - Einige ausgewählte Stäcke aus Clemens übersetzte R. Ornsby, The Month 19 (1873). - Joseph B. Mayor begleitete seine Ausgabe von Strom. VII mit einer englischen Übersetzung London 1902.

\title{
F. Bemerkungen zu der vorliegenden Ausgabe.
}

Die erste Anregung zur Beschäftigung mit Clemens Alex. erhielt ich im Jahre 1888 durch Herrn Geheimrat Dr. Iwan von Müller, damals Professor in Erlangen, der mir riet, eine Arbeit über diesen Autor für das philologische Specialexamen einzureichen und mich nicht nur bei Anfertigung jener Arbeit (sie wurde als Doctordissertation gedruckt: 
Observ. crit. in Clem. Alex. Erlangen 1890) mit wertvollem Rat unterstützte, sondern auch weiterhin meinen Arbeiten die freundlichste Teilnahme zeigte. Im Frühjahr 1895 kam dann durch Herrn Professor D. Harnack die Anfrage an mich, ob ich die Bearbeitung des Clem. Alex. für die Berliner Kirchenväterausgabe übernehmen wolle. Ein zweimaliger Urlaub, der mir vom Kgl. Staatsministerium des Innern für Kirchen- und Schulangelegenheiten in den Schuljahren 1895/96 und 1901/02 gewährt wurde, ermöglichte es mir, die HSS in Paris, Modena und Florenz selbst zu collationieren und einige Zeit ausschließlich der Vorbereitung der Ausgabe zu widmen, während in anderen Jahren die Tätigkeit in der Schule nur wenig Zeit dafür übrig ließ. Eine besondere Förderung erhielt meine Arbeit dadurch, daß ich im Oktober 1902 nach München versetzt wurde und so die reichen Sammlungen der K. Hofund Staatsbibliotkek und der K. Universitätsbibliothek bequem benutzen konnte. Den Vorständen und Beamten dieser Bibliotheken sei auch an dieser Stelle für alle Hilfe, die sie mir gewährten, geziemend gedankt.

Es war mir möglich, einiges ungedruckte Material zu benutzen. Durch Vermittlung von Herrn Geheimrat Hartwig in Halle erhielt ich die in der dortigen Universitätsbibliothek aufbewahrten Vorarbeiten Theodor Heyses für die von ihm geplante Clemensausgabe. Diese Vorarbeiten sind: 1. Collation von Strom. I-VIII (Editio princ.) mit Laur. V 3; 2. Collation von Paed. I-III (Editio princ.) mit Laur. V 24; 3. Bemerkungen zu diesen Collationen; 4. Bemerkungen zu Protr. mit der Überschrift "Revision des Textes in der Ausgabe von Klotz ohne Benutzung anderweitiger literarischer Hilfsmittel oder gar Vergleichung irgend einer Handsehrift"; 5. Bemerkungen zu Paed. I-III nach der Ausgabe von Klotz mit Nachträgen nach Benutzung der Ausgabe Dindorfs; 6. Reinschrift der Bemerkungen zu Paed. I-II 80.

Die Collationen Heyses ergaben sich mir bei Nachprüfung als sorgfältig und dienten mir zur Controlle der eigenen Collation. Die Bemerkungen enthalten sehr viele Conjecturen; viele davon weichen $\mathrm{zu}$ sehr vom überlieferten Texte $a b$, manche aber scheinen mir den Text richtig herzustellen. Vortrefflich ist z. B. Strom. VIII 22 hergestellt

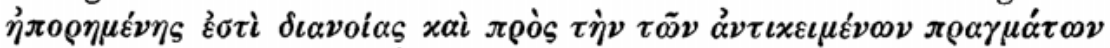

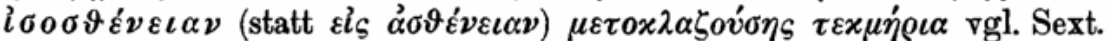

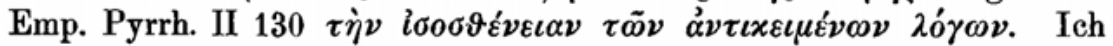
habe im Apparat eine Auswahl aus den Conjecturen Heyses aufgefübrt.

Durch die Güte von Herrn Professor Dr. K. J. Neumann konnte ich auch die Vorarbeiten Eduard Hillers für meine Ausgabe verwerten. Es sind dies 307 halbstand beschriebene Quartseiten mit fortlaufenden Bemerkungen zu Strom. I 1 bis V 27. Sie zeigen, wie sorg- 
fältig sich Hiller um das Verständnis des Textes bemüht hat, und waren mir besonders wertvoll durch Literaturnachweise. Emendationen enthalten diese Seiten weniger als die Notizen Heyses. Immerhin sind auch von ihm manche Stellen richtig hergestellt worden.

Durch die Freundlichkeit von Herrn Professor G. Knaack in Stettin konnte ich auch das in seinen Besitz übergegangene Handexemplar von J. Bernays benutzen, dessen Randbemerkungen in manchen Fällen Belehrung spendeten.

Zu besonderem Danke bin ich Herrn Professor Joseph B. Mayor in Kingston Hill (Surrey) verpflichtet. Die kritischen Bemerkungen zu Protr., Philogus 58 (1898) S. 266-280, und zu Strom. I-VII, Class. Review 8 (1894) S. 233-239; 281-288; 385-391; 9 (1895) S. 97-105; $202-206 ; 297-302 ; 337-342 ; 385-390 ; 433-439$ sind die wertvollsten Beiträge zur Textkritik unseres Autors, die seit der Ausgabe Sylburgs erschienen sind. Kein Herausgeber hat solche Mühe darauf verwendet, den vorliegenden Text zu verstehen oder zu verbessern wie er. Prof. Mayor hat mir aber außerdem noch eine große Menge von Verbesserungen, Quellennachweisen und Bemerkungen über die Interpunction zu Protr. und Paed. brieflich zur Verfügung gestellt. Konnte ich auch nicht alle Conjecturen in den Text aufnehmen, so werden die Benützer der Ausgabe doch Mayors Namen fast auf jeder Seite finden. Schließlich hat Prof. Mayor mir auf meine Bitte noch kurz vor Abschluß meiner Arbeit die Bemerkungen von Jer. Markland (geb. 1693 gest. 1776) zugesandt, die derselbe am Rande seines Exemplars der Potterschen Ausgabe (jetzt im Britischen Museum unter der Signatur 3670. e.) notiert hatte. Es sind dies eine große Anzahl von Conjecturen, darunter manche recht einleuchtende Verbesserungen. Eine Auswahl davon ist im Apparat mitgeteilt.

Zu Dank bin ich ferner verpflichtet Herrn Professor Henry Jackson ${ }^{1}$ in Cambridge, der mir eine Anzahl wichtiger Parallelstellen aus den griechischen Philosophen mitteilte, Herrn Professor George F. Mo ore in Andover Mass., der mir die Randbemerkungen P. de Lagardes (die allerdings wenig Neues boten) aus dessen Clemensausgaben in der Universitätsbibliothek der Stadt New-York abschrieb, und nicht zum wenigsten Herrn Bibliothekar Dr. R. Münzel in Hamburg, der mir wertvolle Ergänzungen zu seinem Artikel über den Paed. (Beiträge zur Bücherkunde u. Philologie, August Wilmanns zum 25. März 1903 gewidmet S. 293-300), wichtige Parallelstellen, besonders aus Plato und

1) Vgl. auch seine Artikel im Journal of Philol. 24 (1896) S. 263-271; 27 (1899) S. 136-144; 28 (1900) S. 131-134. 
Lucian, mitteilte und mich auf die Benutzung des Clemens durch Isidor von Pelusium aufmerksam machte.

Während des Druckes hatte Herr Professor C. Weyman die Freundlichkeit, mich auf manche Literaturangabe oder Parallelstelle aufmerksam zu machen, und Herr Professor W. Kroll, der ebenfalls einen Teil der Druckbogen mitzulesen die Gute hatte, gab manchen wertvollen Beitrag zur Emendation. Vor allem aber habe ich den.Herrn Professoren E. Schwartz u. U. von Wilamowitz-Moellendorff zu danken, die alle Druckbogen aufs genaueste durchsahen und mir ihre Verbesserungen zusandten. Beider Name findet sich oft genug im Apparat; doch gibt das nur eine sehr ungenuggende Vorstellung von der mannigfachen Hilfe, die sie meiner Arbeit zuteil werden ließen: mancher Fehler im Text, manche falsche Conjectur im Apparat wurde beseitigt, die Interpunction an vielen Stellen gebessert; mancher Druckfehler wäre ohne ihre Mitarbeit stehen geblieben.

So ist es vielfach fremde Arbeit, die der Ausgabe zugute gekommen ist. Ihr ist es nicht am wenigsten zu verdanken, wenn die Ausgabe brauchbar geworden ist. Allen, die dazu geholfen, gebührt also nicht nur der Dank des Herausgebers, sondern auch der des Benutzers der Ausgabe.

Die Seitenzahlen der Ausgaben Sylburgs und Potters stehen mit $\mathrm{S}$ und $\mathrm{P}$ am Rand, eine Tabelle mit den Seiten der Pariser Ausgabe (1629) wird am Schluß der Ausgabe beigegeben werden, da nach ihr noch immer häufig citiert wird. Die Paragrapheneinteilung von Klotz habe ich trotz ihrer Mängel beibehalten, um nicht die Verwirrung (oft wird Clemens in einem Buche auf dreierlei Art citiert) noch größer zu machen. Doch habe ich die großen Paragraphen in Unterabschnitte zerlegt. In der Ausgabe selbst ist stets darnach (mit Weglassung der Capitelzahlen) citiert. Die Zahlen bei den Verbesserungen Cobets verweisen auf die Seiten von Logios Hermes I (1866. 1867). Historikerfragmente sind nach C. Müller, FHG, Komikerfragmente nach Th. Kock, CAF, Lyrikerfragmente nach Th. Bergk, PLG ${ }^{4}$, Tragikerfragmente nach A. Nauck, TGF² ohne nähere Angabe citiert.

München, Dezember 1904.

Otto Stählin. 
